\title{
EL CURRÍCULO DE MATEMÁTICAS DESDE LA CULTURA INDÍGENA: UNA REVISIÓN DEL ESTADO DEL ARTE ${ }^{1}$
}

\author{
THE MATH CURRICULUM FROM INDIGENOUS CULTURE: A REVIEW OF THE \\ STATE OF THE ART
}

\section{O CURRÍCULO DE MATEMÁTICA DA CULTURA INDÍGENA: UMA REVISÃO DO ESTADO DA ARTE}

\author{
Edwin Molano Franco; Hilbert Blanco Álvarez
}

\begin{abstract}
Magister en Enseñanza de las Ciencias Exactas y Naturales, Universidad Nacional de Colombia. Docente de matemáticas, Colegio Sagrado Corazón de Jesús. ORCiD: 0000-0001-6281-8949. E-mail: edwinmofr@unisabana.edu.co, Colombia.

Doctor en Ciencias de la Educación, Universidad de Granada, España. Docente del Departamento de Matemáticas y Estadística, Universidad de Nariño. ORCiD: 0000-0003-4973-8076. E-mail: hilbla@udenar.edu.co, Colombia.
\end{abstract}

Recibido: 10 de diciembre de 2021

Aprobado: 16 de diciembre de 2021

DOI: https://doi.org/10.22267/rtend.222301.196

\footnotetext{
${ }^{1}$ Este artículo corresponde a los resultados parciales de una investigación doctoral que busca identificar y caracterizar elementos para la construcción de una política educativa emancipatoria desde el diálogo entre el saber matemático y la cultura indígena a través de la Etnomatemática, del doctorado en educación de la Universidad de La Sabana.
} 


\title{
Resumen
}

El objetivo de este artículo es contribuir al conocimiento del estado de desarrollo de las investigaciones sobre la incidencia de la cultura indígena en el currículo de matemáticas desde una perspectiva política. Esta investigación es de tipo documental desarrollada con metodología cualitativa e interpretativa, la cual hizo uso del análisis de contenido con el software Atlas.ti. y la pregunta orientadora fue ¿de qué manera la cultura indígena incide en el nivel de los principios normativos, de las instituciones y de la praxis política a través del currículo? El material empírico fueron artículos, monografías de pregrado, trabajos de maestría y tesis doctorales, publicadas entre el año 2010 y 2020. Los resultados se presentan en tres niveles: en el nivel de los principios éticos se plantea el reconocimiento y respeto a la diferencia; en el nivel institucional se plantea la flexibilización del currículo para vincular otros saberes y prácticas, incluir a la comunidad, así como la reflexionar sobre valores, creencias y saberes; y en el nivel de la praxis política se propone la insubordinación creativa.

Palabras clave: colonialismo; cultura; educación; enseñanza de las matemáticas; política educacional.

JEL: I20, I21

\begin{abstract}
The objective of this article is to contribute to the knowledge of the state of development of research on the incidence of indigenous culture in the mathematics curriculum from a political perspective. This research is of a documentary type developed with qualitative and interpretive methodology, which made use of content analysis with the Atlas.ti software. and the guiding question was in what way does indigenous culture affect the level of normative principles, institutions, and political praxis through the curriculum? The empirical material consisted of articles, undergraduate monographs, master's works, and doctoral theses, published between 2010 and 2020. The results are presented on three levels: at the level of ethical principles, recognition and respect for difference are proposed; At the institutional level, the flexibility of the curriculum is proposed to link other knowledge and practices, including the community, as well as reflect on values, beliefs and knowledge; and at the level of political praxis creative insubordination is proposed.
\end{abstract}


Keywords: colonialism; culture; education; mathematics education; educational policy. JEL: I20, I21.

\section{Resumo}

O objetivo deste artigo é contribuir para o conhecimento do estado de desenvolvimento das pesquisas sobre a incidência da cultura indígena no currículo de matemática, de uma perspectiva política. Esta pesquisa é do tipo documental desenvolvida com metodologia qualitativa e interpretativa, que utilizou a análise de conteúdo com o software Atlas.ti. e a questão norteadora foi de que forma a cultura indígena afeta o nível de princípios normativos, instituições e práxis política por meio do currículo? O material empírico foi constituído por artigos, monografias de graduação, trabalhos de mestrado e teses de doutorado, publicados entre 2010 e 2020. Os resultados são apresentados em três níveis: ao nível dos princípios éticos propõe-se o reconhecimento e o respeito pela diferença; No nível institucional, a flexibilização do currículo é proposta para articular outros saberes e práticas, incluir a comunidade, bem como refletir sobre valores, crenças e saberes; e no nível da práxis política a insubordinação criativa é proposta.

Palavras-chave: colonialismo; cultura; educação; educação matemática; política educacional. JEL: I20, I21.

\section{Introducción}

En el marco de una investigación doctoral ${ }^{2}$ que busca Identificar y caracterizar elementos para la construcción de una política educativa descolonizadora desde el diálogo entre el saber matemático y la cultura indígena a través de la Etnomatemática, se plantea esta investigación documental en la que se analiza los cuestionamientos y propuestas que se formulan al currículo de matemáticas desde la cultura indígena, a partir de las diferentes aproximaciones que han hecho los investigadores y los pueblos indígenas en la última década desde una perspectiva Etnomatemática, por medio de un análisis que toma como base la estructura arquitectónica de la política de Dussel

\footnotetext{
${ }^{2}$ Investigación doctoral titulada: Elementos para una política emancipatoria de educación matemática: un diálogo entre la cultura indígena y la Etnomatemática, dirigida por el doctor Hilbert Blanco-Álvarez en el marco del Doctorado en Educación de la Universidad de La Sabana, Chía, Colombia.
} 
(2009) en la que se mostrará como la cultura incide en los tres niveles de la política (principios normativos, institucional, y de la acción estratégica) a través del currículo.

Para el análisis, se utilizaron las categorías construidas previamente a partir de las investigaciones que han trabajado la relación Educación Indígena - Etnomatemática - Currículo (Molano y Blanco, 2021), y que aquí se describen de manera breve.

Se hará una descripción metodológica de la manera como se trató el material empírico, después se plantea de manera breve el contexto de transformación de la educación indígena, seguidamente se realiza el análisis de la transformación curricular y su incidencia en la política de educación matemática, y finalmente, se presentan las conclusiones.

\section{Material empírico y métodos de búsqueda}

La recolección del material empírico se centró en: artículos, trabajos de pregrado, maestría y tesis doctorales, publicadas o realizadas entre el año 2010 y 2020. La búsqueda de éstos se realizó en las bases de datos Scopus, Web of science, Academic Search Complete, Fuente Académica Premier, Jstore, ProQuest, Tylor and Francis, Redalyc y Google Académico. Los términos clave fueron educación indígena, etnomatemáticas y currículo, y las ecuaciones de búsqueda fueron (ethnomathemat* or ethnomatemat* or etnomatematic* or "mathematics culturally") AND (curricul* or "curriculum development" or "curriculum design" or "curriculum and instruction") AND (indigenous or indigena* or "indigenous people" or native or aboriginal or Indians or "first nations"); y sus respectivas traducciones al español y portugués.

\section{Metodología}

La metodología empleada fue el análisis cualitativo e interpretativo de contenido, y se realizó en tres etapas:

Etapa 1: Codificación de enunciados relativos a cambios curriculares por medio de indicadores. Esto se realizó haciendo uso del software Atlas.ti. versión 6.0. en un proceso de lectura en 
profundidad del material y de codificación constante de la información de acuerdo con los indicadores presentados en la Tabla 1.

\section{Tabla 1}

Indicadores para la codificación de las unidades de información.

Indicadores Descripción

Problematizaciones Se refiere a cuestionamientos y críticas al currículo de matemáticas.

Propósitos Se refiere a intensiones, objetivos, intereses que vinculados a la transformación curricular en matemáticas.

Propuestas Se refiere a aquellas enunciaciones que señalan un camino a seguir para realizar las transformaciones curriculares que se plantean.

Advertencias $\quad$ Se refiere a aquellos obstáculos y tensiones con los que es posible encontrarse al plantear los cambios curriculares.

Fuente: Producción propia.

Etapa 2: Interpretación del material codificado en relación a las categorías de análisis. En esta etapa se realizó un apareamiento entre las enunciaciones clasificadas de acuerdo a cada uno de los indicadores de la Tabla 1, y las categorías y subcategorías planteadas como herramientas de análisis que se muestran en la Tabla 2, las cuales fueron construidas previamente y cuyo desarrollo puede leerse en profundidad en Molano y Blanco (2021).

\section{Tabla 2}

Dimensiones, categorías, y subcategorías utilizadas en el análisis.

\begin{tabular}{|c|c|c|c|}
\hline $\begin{array}{l}\text { Dimensiones } \\
\text { emergentes }\end{array}$ & $\begin{array}{l}\text { Categorías } \\
\text { emergentes }\end{array}$ & $\begin{array}{l}\text { Subcategorías } \\
\text { emergentes }\end{array}$ & Descripción \\
\hline & Relaciones de poder & Colonialismo & $\begin{array}{l}\text { Hace referencia a procesos de } \\
\text { dominación a través del control, la } \\
\text { exclusión y el ocultamiento. }\end{array}$ \\
\hline Social & & Emancipación & $\begin{array}{l}\text { Se refiere a procesos de resistencia } \\
\text { ante el colonialismo a través de la } \\
\text { reivindicación, la criticidad, la } \\
\text { promoción social y la excelencia } \\
\text { escolar. }\end{array}$ \\
\hline
\end{tabular}




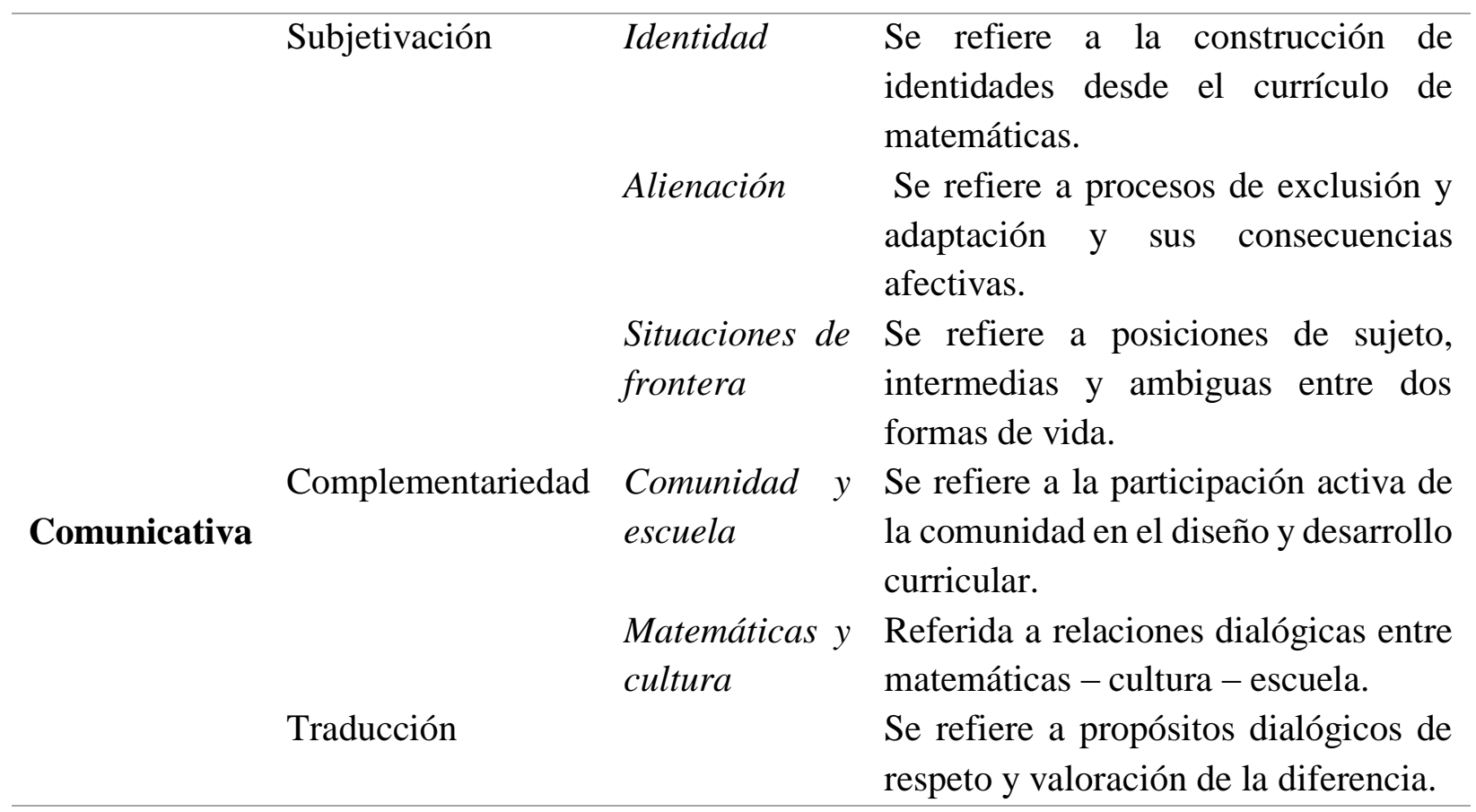

Fuente: Producción propia.

Etapa 3: Construcción discursiva de los resultados obtenidos. En esta etapa se realizó una interpretación de los datos categorizados en relación a la estructura arquitectónica de la política propuesta por Dussel (2009), los cuales se presentan en el siguiente apartado.

\section{Incidencia de la cultura indígena en el currículo de matemáticas}

Se inicia señalando algunos elementos contextuales sobre los que se vienen dando transformaciones en las escuelas indígenas en Colombia.

Las políticas de educación indígena en Colombia al igual que en muchos otros países de Latinoamérica y el mundo, se ha guiado bajo un interés colonizador, es decir como una forma del ejercicio de la dominación sobre los pueblos indígenas para imponer la cultura de los invasores, donde el saber matemático, desde una concepción platónica, ha servido para el ocultamiento y exclusión de la diversidad cultural, generando problemas de discriminación y alfabetización matemática (Aikenhead, 2017; Peña et al., 2017). Dicho proceso colonizador, por supuesto, ha encontrado resistencias tanto en los mismos pueblos indígenas a través de sus organizaciones, como de algunos académicos que desde una posición crítica cuestionan la racionalidad moderna, 
sometida a las reglas del mercado. Así, en las últimas décadas ha venido tomando fuerza la perspectiva sociocultural de la educación matemática (Radford, 2013), desde la cual la Etnomatemática se preocupa por reivindicar otras racionalidades y establecer mecanismos dialógicos entre el saber matemático cultural y la matemática escolar o formal.

Por otro lado, en Colombia los pueblos indígenas llevan varias décadas resistiendo a dicha forma de colonialismo y reclamando derechos políticos de reconocimiento y autonomía, desde organizaciones como el Consejo Regional Indígena del Cauca (CRIC) y la Organización Nacional Indígena de Colombia (ONIC), para lo cual la educación escolar se plantea como herramienta de emancipación y fortalecimiento identitario y cultural (Arbelaez Jiménez \& Vélez Posada, 2008; Hammen, 1992; Londoño Sulkin, 2003).

Es así como en el ámbito nacional, desde el Sistema Educativo Indígena Propio (SEIP), se viene trabajando en la construcción de propuestas para una educación indígena para "Garantizar el derecho fundamental a la educación propia en el marco de la diversidad de culturas, para lograr la pervivencia cultural de los pueblos indígenas en Colombia” (CONTCEPI, 2013, p. 53), y otras propuestas más regionales como las de la Sierra Nevada de Santa Marta (Peña et al., 2017), el departamento del Cauca (Tamayo, 2017), o en el departamento del Amazonas (Asociación PANI, 2018), por nombrar algunas, con diferentes niveles de desarrollo, y que desde una posición crítica cuestionan tanto el saber matemático como la misma escuela.

Estos señalamientos nos muestran el carácter dinámico de la política, en la que los pueblos indígenas vienen presentando oposiciones constructivas. En este sentido, investigadores como Rojas y Castillo (2005) han mostrado que la diversidad cultural ha servido como discurso para representar a ciertos grupos sociales y legitimar las políticas educativas estatales, pero también como lugar de posicionamiento político desde el que los grupos subalternizados han logrado poner en la agenda política una serie de demandas e incidir en las decisiones estatales. En esta misma línea Cárdenas y Urueta (2014) realizan un análisis la manera en que la diversidad cultural se instaló en la educación pública colombiana y ha devenido en un saber escolar. 
Para Cárdenas y Urueta (2014), la emergencia de la cultura en la educación pública colombiana se da a partir de pactos internacionales a mediados del siglo XX que asignan gran importancia a ideas como la no exclusión, y en tal sentido se empiezan a reconocer derechos individuales y colectivos; el alumno se concibe como un individuo que debe ser atendido; aparecen nuevos modelos educativos; y toman fuerza los movimientos indígenas. De tal manera que la cultura emerge a través de reconocimientos legislativos, es decir, a través de acciones sobre el nivel institucional. A partir de los lineamientos generales de educación indígena publicados por el Ministerio de Educación Nacional en 1982, la cultura se convierte en el marco referencial de todas las acciones de la etnoeducación, incidiendo en los principios de la política, construyendo estrategias y determinando los manejos administrativos. La diversidad se convierte en saber escolar que genera nuevos saberes que inciden en el fortalecimiento de las subjetividades y conocimientos.

En este orden de ideas, se pueden señalar al menos tres ámbitos en los que se viene avanzando en transformaciones curriculares que atiendan a las necesidades de las escuelas indígenas. Primero, en la legislación colombiana, se han reconocido derechos de los pueblos indígenas como sujetos políticos, y se han abierto las puertas para la incorporación de nuevas prácticas y conocimientos matemáticos en el currículo (Peña et al., 2017); segundo, en la última década se ha dado un aumento considerable en la cantidad de investigaciones que se plantean transformaciones curriculares en relación con la cultura indígena desde la perspectiva etnomatemática, en las que se buscan nuevas comprensiones y sentidos de la educación matemática, las cuales constituyen la documentación analizada en el presente estudio, como puede verse en la Figura 1; tercero, las investigaciones señalan la posibilidad de establecer vínculos dialógicos desde la complementariedad, que permitan la sobrevivencia y trascendencia de las culturas indígenas, a la vez que se alcance la excelencia escolar, como se mostrará a lo largo de este artículo. 


\section{Figura 1}

Producción académica por año sobre la relación Educación Indígena - Currículo Etnomatemática.

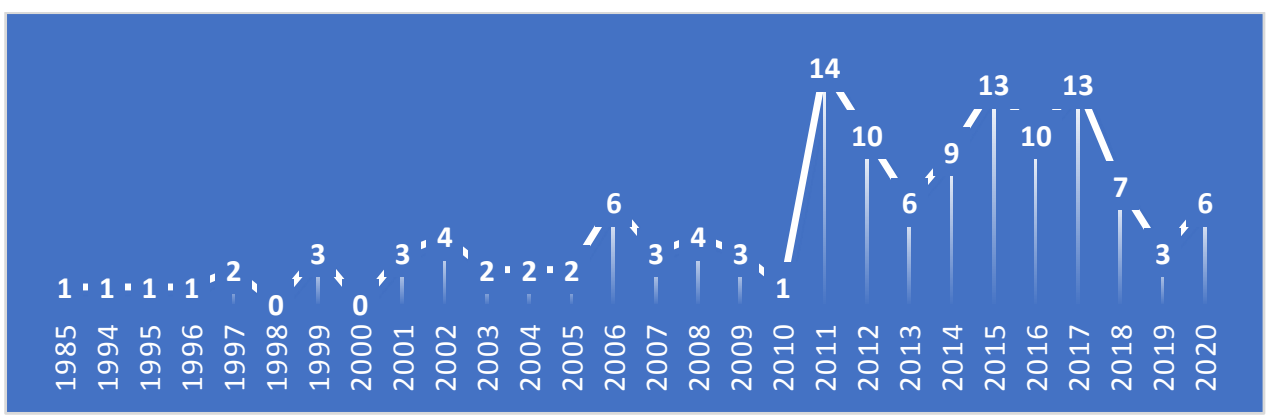

Fuente: Producción propia.

\section{Análisis}

Tomando como base la arquitectura de la política propuesta por Dussel (2009), se analizará la manera en que desde una perspectiva Etnomatemática, la cultura indígena cuestiona el currículo de matemáticas como institución objetiva de la política educativa, a la vez que propone transformaciones acordes con las demandas y propósitos de los pueblos indígenas.

\section{Definiciones Previas}

Enrique Dussel (2009), plantea una arquitectura de la política compuesta por tres niveles como se muestra en la Figura 2 que se usará para realizar este análisis.

\section{Figura 2}

Niveles arquitectónicos de la política.

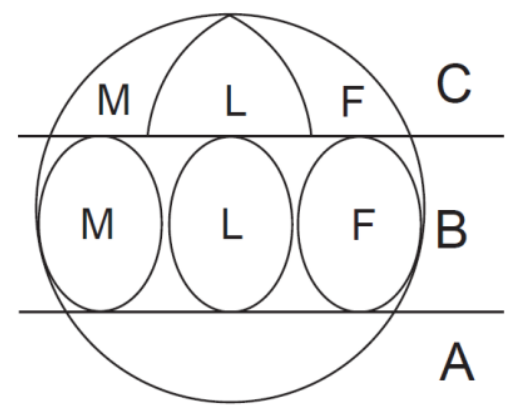

Fuente: (Dussel, 2009, p. 43). 
El nivel C, se refiere a los principios normativos de la política. El nivel B, se refiere a la institucionalidad. El nivel A, se refiere a la acción estratégica o praxis política. En los niveles $\mathrm{C}$ y B se dan tres esferas M: material, L: legitimidad, y F: factibilidad. Por ahora el análisis se centrará en las tres esferas del nivel institucional.

La esfera material de la política se refiere a aquellas instituciones que por su contenido se constituyen en la "materia" de la política, es decir aquellas instituciones cuyo contenido es necesario para la afirmación y promoción de la vida que es la "materia" o el asunto de la política. A esta esfera material pertenecen campos como la economía, la ecología, la educación, la cultura, entre otros.

La esfera de legitimidad se refiere a las instituciones que organizan de manera formal las instituciones materiales y de factibilidad, a esta esfera pertenecen las instituciones democráticas de participación, de validación, y de decisión.

La esfera de factibilidad, se refiere a las instituciones que objetivan las instituciones materiales y de legitimidad, haciendo posible los principios postulados por la comunidad política.

Desde esta arquitectónica de la política propuesta por Dussel (2009), se podrían leer otras investigaciones como la de Cárdenas y Urueta (2014), que señalamos más arriba, en la que se identifica la influencia de la cultura indígena en la educación pública colombiana en sus tres niveles así:

En el nivel de los principios normativos:

- Igualdad ante la ley.

- La diversidad como constitutiva de los pueblos.

- El territorio como fundamento de la vida y la educación. 
En el nivel institucional:

- Generación de normas para el reconocimiento de la diversidad y su objetivación.

- Creación de herramientas como libros y lineamientos para la planificación.

- Creación de instituciones como el grupo de Etnoeducación y asociaciones indígenas.

- Ampliación de programas de escolarización.

En el nivel de la praxis:

- El reconocimiento de la cultura implica la participación de la comunidad y responsabilización sobre el diseño e implementación de la acción educativa.

- El cambio en la escuela genera nuevas subjetividades.

- Emergencia de la cultura como campo desde el que se sitúa el indígena como actor político.

Para esta investigación, el foco se situó en el currículo de matemáticas para analizar la manera en que la cultura indígena, desde una perspectiva Etnomatemática, cuestiona y plantea transformaciones que inciden en los tres niveles de la política.

\section{Currículo como institución de factibilidad}

El currículo, por su raíz latina Scurrere (correr) es relacionado con una ruta o camino a seguir, a través del cual se transmite y ejecuta un propósito educativo (Costa, 2009), es decir que la selección de contenidos, metodologías y relaciones, que se establecen en el currículo, está determinada por relaciones de poder, tensiones y conflictos de orden político, social y económico (Fuentes, 2014b; Tamayo, 2012), llegando a funcionar como herramienta de control a través de una distribución y organización de los saberes, que los jerarquiza, los clasifica y les asigna valores (Monteiro y Rodrigues, 2011). En tal sentido, Aikenhead (2017) señala que desde que se crearon los primeros currículos de matemáticas para escuelas públicas en el siglo XIX, con base en el pensamiento socrático, platónico y aristotélico, se instauró de manera arbitraria la relación entre matemática escolar y discurso formal. 
Si bien, no existe una definición precisa y concertada de lo que es el currículo, es posible clasificar las posturas de los investigadores bajo alguna o varias de las siguientes categorías: como espacio, como proceso, como conjunto de elementos, o como herramienta. En tal sentido, el currículo es una herramienta para la constitución de subjetividades e identidades, compuesta por un conjunto de elementos referidos al plan de estudios, las relaciones interpersonales, y la estructuración espacio-temporal, articulados en un espacio de relaciones de poder, en un proceso de reconstrucción y resignificación permanente en torno a los intereses de los sujetos que participan de él.

De lo anterior, podemos decir que el currículo moviliza los elementos de la esfera material como el saber matemático, de acuerdo con un orden de legitimidad y de formalidad atendiendo a principios que sustentan los intereses de los actores políticos, por lo que se considera al currículo como institución de factibilidad.

\section{La matemática como institución material}

En la literatura relacionada se hace referencia a la Matemática institucionalizada, adjetivándola como occidental, académica, disciplinar, dominante o formal. Para este análisis, se llamará simplemente Matemática, con M mayúscula. A esta Matemática se la define principalmente desde una concepción formalista, como un lenguaje o tecnología simbólica (Gavarrete, 2012), un cuerpo de conocimientos, de datos, algoritmos, axiomas y teoremas (Rosa y Orey, 2011), un conjunto de disciplinas y una actividad científica (Villavicencio, 2011), que generaliza, abstrae y plantea escenarios hipotéticos (Parra, 2003). Para D’Ambrosio (2011, citado en Higuita, 2014) es la ciencia que está relacionada con los números, las formas, relaciones, medidas e inferencias.

Ahora bien, si se concibe a las matemáticas como un conjunto de prácticas, cursos operatorios, a la manera del materialismo formalista (Madrid, 2009), en la que los sujetos actúan coherentemente con un universo discursivo, mediante "procesos (...) realizados sobre objetos (...) y/o proposiciones" (p. 36), podría decirse que las matemáticas son una institución como es definida por Diaz y Batanero (1994). 
Una institución (I) está constituida por las personas involucradas en una misma clase de situaciones problemáticas. El compromiso mutuo con la misma problemática conlleva la realización de unas prácticas sociales compartidas, las cuales están, asimismo, ligadas a la institución a cuya caracterización contribuyen. (p. 9)

De esta manera, el saber matemático, ahora como institución, queda ubicado en la esfera material en tanto que aquellas prácticas, objetos y problemáticas de las que tratan, contribuyen o son empleadas políticamente en relación a la reproducción y desarrollo de la vida de la comunidad política $^{3}$, pues responden a la satisfacción de ciertas necesidades como podría ser la distribución de alimentos, de territorios, o la construcción de casas y herramientas, al desarrollo científico y tecnológico, entre muchas otras.

\section{Momento crítico del campo cultural sobre el currículo}

La Etnomatemática en coherencia con las demandas de los pueblos indígenas, pone el énfasis en la relación del campo cultural con la educación matemática, y de esta forma, desde las subcategorías colonialismo y alienación, se señalan cuestionamientos al currículo en relación a los tres niveles arquitectónicos de la política de la siguiente manera:

Respecto al nivel de los principios normativos, se cuestiona el establecimiento de una verdad, una visión de mundo y un proyecto social. El currículo como institución de factibilidad moviliza en la acción estratégica los principios normativos de la política constituidos desde la modernidad y el neoliberalismo. Esa pretensión de una verdad única construida desde la Matemática, es la verdad científica de un sujeto individual, capaz de abstraerse del mundo ubicándose en el "punto cero" (Castro, 2005), es la visión de un mundo que como res-extensa (Descartes, 1641) queda en una posición jerárquicamente inferior que el cogito, dispuesta a la voluntad de poder (como dominación $)^{4}$ del sujeto moderno, en busca de la maximización del rendimiento académico como medida de avance hacia el desarrollo económico.

\footnotetext{
${ }^{3}$ A pesar de que también hayan contribuido a la creación de armas de destrucción, en principio tienen por propósito la afirmación de la vida y no de la muerte.

${ }^{4}$ Una concepción diferente del poder puede leerse en Dussel (2009).
} 
De esta manera, la cultura critica, el eurocentrismo que impone la universalidad de su particularidad, que postula la Matemática como un saber exento de interpretaciones culturales, pero además estático, prediseñado y preexistente, implicando la imposibilidad de la existencia de otros saberes (Fuentes Leal, 2014a; Miarka, 2011; Monteiro \& Rodrigues, 2011; Peña-Rincón et al., 2017; Rosa \& Orey, 2011, 2012; Tutak et al., 2011), representando al indígena por los valores opuestos (local, contextualizado, ideológico, no objetivo, abarcador de múltiples verdades) con lo que termina excluyéndolo y posicionándolo en un pasado desde la falsa dicotomía modernidad/tradición.

Respecto al nivel institucional, el currículo como institución de factibilidad, determina la esfera material por su posibilidad de realización empírica administrando el saber matemático, mientras que dispone un conjunto de prácticas y mecanismos para atender a la esfera de legitimidad por medio de la validación de las prácticas desde unos estándares o lineamientos y unas pruebas estandarizadas.

En tal sentido, se cuestionan la distribución, organización y legitimación del saber matemático (Monteiro \& Rodrigues, 2011), pues la cultura indígena concibe al conocimiento de manera holística, donde lo curricular no se entendería como un camino o una secuencia de contenidos, sino con un tejido en el que cada hilo se vincula con todos los demás en una interrelación permanente y necesaria, de tal manera que el aprendizaje, por ejemplo, de los casos de factorización, descontextualizado y desvinculado de otros saberes, contenidos y problemáticas, pierde sentido en la relación entre el campo cultural y la política educativa.

Respecto al nivel de la praxis política, se cuestiona la justificación de la educación matemática respecto al entrenamiento de la mente y la formación de ciudadanos y buenos trabajadores (característica antropológica de la modernidad), la competencia económica de las naciones y la competitividad laboral (característica económica de la modernidad), enseñanza de conceptos y proposiciones obsoletas, el empoderamiento, la herencia cultural y la justicia social (característica cultural de la modernidad) (Aikenhead, 2017; Aroca, 2016). 


\section{Momento transformador propositivo del campo cultural sobre el currículo}

En el apartado anterior se vio como el campo cultural, como esfera material de la política, en un primer momento (negativo) cuestiona a través del currículo el orden establecido de la política educativa en sus tres niveles. Ahora bien, en un segundo momento (positivo), se proponen cambios curriculares en orden a atender nuevas necesidades y propósitos que surgen del reconocimiento de la problemática presentada desde el campo cultural.

En el nivel de los principios normativos, desde la subcategoría emancipación se plantea la problematización del saber matemático, entendiendo la capacidad colonizadora que tiene la Matemática, exponiendo y denunciando las relaciones de poder entre los saberes escolares y extraescolares (Costa, 2012; Fuentes, 2014b; Jaramillo, 2011; Monteiro \& Rodrigues, 2011; Parra, 2003; Tutak et al., 2011); con el fin de evidenciar otras racionalidades, ordenes o epistemologías (Tamayo, 2017), desde una práctica deconstructiva de la visión eurocéntrica del conocimiento.

Si bien se plantean varios principios para el currículo de matemáticas en escuelas indígenas, como la flexibilidad, interculturalidad, autonomía, interacción, cooperación, multimatemáticas, entre otros (Higuita, 2014; Pinxten \& François, 2011; Tamayo, 2012, 2017); el respeto por la diferencia es mencionado en casi la totalidad de las investigaciones, lo cual implica la inclusión social y el reconocimiento del Otro en su forma particular de pensar matemáticamente, desde sus propias lógicas y dinámicas, pero además, teniendo en cuenta el relacionamiento del indígena con la Madre Tierra, se deriva el respeto por la Naturaleza como fundamento principal (Avendaño et al., 2016; Peña et al., 2017; Shockey, 2017; Stathopoulou \& Appelbaum, 2017; Tamayo, 2016).

Estas consideraciones, suponen la redefinición del tipo de sujeto que se quiere formar, se busca un estudiante reflexivo, crítico y autónomo, lo cual implica la problematización del saber, la elaboración de significados y la vinculación de la perspectiva indígena (Aikenhead, 2017; Alves, 2017; Blanco, 2017; Monteiro \& Rodrigues, 2011).

En el nivel institucional, respecto a la subcategoría complementariedad se plantea la flexibilización del currículo para la formación de identidades y subjetividades, así como para la 
reflexión sobre valores, creencias y saberes (Aikenhead, 2017; Lacerda et al., 2017; Monteiro \& Rodrigues, 2014).

Se proponen cambios en la temporalidad, transformando el calendario y el ritmo de las actividades escolares, en orden a hacer de las prácticas culturales y los espacios-tiempos de la educación ancestral parte de la organización curricular, al integrar las dinámicas ecológicas del entorno natural y los rituales, como una forma de resistencia en la que un pueblo indígena lucha por sobrevivir como cultura (Pereira et al., 2015; Tamayo, 2016, 2017), lo cual implica que la escuela esté dispuesta a reconocer las prácticas sociales, en las que se construyen significados que se negocian a través de acuerdos y refutaciones (Avendaño et al., 2016; Jaramillo, 2011; Weldeana, 2016) y en base a esto, los planes de estudio, deberían poner el foco en los sujetos que se quieren formar, en lugar de unos contenidos preestablecidos (Avendaño et al., 2016; Tamayo, 2012, 2017), pero además se requiere de la participación de la comunidad en el desarrollo y planeación curricular, y poner en permanente relación a las matemáticas con la cosmovisión, la vida diaria y otras ciencias (Blanco, 2017).

En el nivel de la praxis política, desde las categorías de complementariedad y traducción se hace referencia a las prácticas de enseñanza, el campo cultural implica la reflexión y la concienciación de la existencia de unas intencionalidades inherentes a dicha práctica, buscando deslegitimar la violencia y la exclusión (Aroca, 2016), promover nuevas comprensiones (Tamayo, 2012) e "identificar otras formas de producción de conocimientos y saberes coherentes con las apuestas políticas y comunitarias del ser indígenas” (Avendaño et al., 2016, p. 95). En tal sentido, Blanco; Fernández y Oliveras (2017), basados en D’Ambrosio y Espasandin (2015), propone la insubordinación creativa, entendida como una acción deliberada en contra de una regla o régimen educativo establecido, frente a la ruptura de la escuela con el entorno cotidiano del estudiante, donde el aprendizaje situado juegue un papel preponderante.

Ahora bien, la escuela debe constituirse en un espacio de hibridación cultural, donde se propicie la emergencia de nuevas racionalidades, nuevas subjetividades, y nuevas matemáticas. Para lo cual, se requiere de la formación de profesores capaces de desarrollar metodologías propias, que 
sean investigadores y que contribuyan a la construcción de un proyecto educativo coherente con su realidad (Dos Santos \& Donizeti, 2011).

También se propone problematizar el diálogo para comprender las implicaciones del lenguaje en la educación matemática y evitar el neocolonialismo, pero también la búsqueda de significados comunes que permitan un diálogo constructivo entre formas diversas de pensamiento matemático, y en este sentido resulta importante la investigación del pensamiento matemático indígena, respecto a lo cual se pueden resaltar los trabajos de Aroca (2007) con la comunidad indígena Ika de la Sierra Nevada de Santa Marta; de Blanco (2009) con los Tule en el golfo de Urabá; de Costa (2009) con los Tikuna en el Amazonas; de Higuita (2014), con los Embera Chamí del suroeste antioqueño; y de Parra y Trinick (2017) con los Nasa del departamento del Cauca, por nombrar algunos.

Finalmente, Blanco (2017a) realiza un aporte teórico-metodológico para examinar la adecuación de los currículos de matemáticas a las necesidades de la educación intercultural, el reconocimiento, valoración y uso de los saberes extraescolares en el aula de clase y las implicaciones afectivas en los estudiantes.

\section{Tensiones y obstáculos para los cambios curriculares en matemáticas en las escuelas indígenas en Colombia}

\section{Tensiones}

En el nivel de los principios normativos, como se ha venido señalando, desde las subcategorías colonialismo y emancipación, el campo cultural pone en tensión los principios que norman las políticas de educación matemática, al cuestionar la homogenización y el mejoramiento de los aprendizajes de unos saberes disciplinarizados desde la modernidad y el neoliberalismo, frente a las propuestas indígenas que reivindican la diversidad epistémica y cultural, y el reconocimiento de la Madre Tierra como fundamento de toda acción. En tal sentido, la escuela indígena y el saber matemático se conciben en un proceso de resignificación, de dispositivo de poder colonial hacia un instrumento político de resistencia a la dominación (Peña et al., 2015; Pereira et al., 2015; Tamayo, 2017). 
En el nivel institucional, desde la subcategoría emancipación, el reconocimiento de la colonialidad del saber, plantea la descolonización y resignificación de la escuela y el saber matemático. Este proceso se ve tensionado por la autonomía de las escuelas para realizar cambios en la organización espacio-temporal fundamentados en la relación con la naturaleza y el desarrollo de la cultura (Avendaño et al., 2016; Higuita, 2014; Tamayo, 2016); para desarrollar currículos propios diferenciados, puesto que aunque documentos oficiales como los Lineamientos Curriculares y los Estándares Básicos de Competencias plantean una concepción socio-cultural de las matemáticas, y existe la autonomía para desarrollar currículos propios, ésta queda limitada en términos de la obligatoriedad de presentar pruebas estandarizadas (Peña \& Blanco, 2015) las cuales establecen fronteras o límites para el surgimiento y desarrollo de nuevos paradigmas en la educación matemática (Blanco et al., 2014), adicionalmente, la ley general de educación (Ministerio de Educación Nacional, 1994) en su artículo 23 obliga la enseñanza de disciplinas establecidas por el estado en un $80 \%$ del plan de estudios, lo cual es considerado como una desigualdad educativa y de oportunidades, por lo que se reclaman adecuaciones curriculares para las escuelas indígenas.

En el nivel de la praxis política, en relación a la categoría subjetivación y la subcategoría situaciones de frontera los profesores se ven tensionados entre prácticas educativas disciplinares e indisciplinares; entre el gobierno del estado y el de sus propias comunidades; entre desarrollar su cultura e identidad y atender a las demandas nacionales (Avendaño et al., 2016; Tamayo, 2016), por lo que se plantea la necesidad del reconocimiento y asunción de la hibridación o mestizaje cultural, donde múltiples formas de percibir el mundo entran en una negociación de significados (Barta y Shockey, 2016; Pereira et al., 2015; Tamayo, 2017).

Por otro lado, como tensiones internas a las comunidades: primero, padres de familia, maestros y autoridades tradicionales pueden dudar de los beneficios de la enseñanza basada en la cultura, o no sentirse reconocidos como participantes activos de las decisiones curriculares (Nutti, 2018; Tamayo, 2017). Segundo, Aikenhead (2017) señala que, aunque se hagan importantes esfuerzos en el aula acerca del reconocimiento de la raíz cultural de las matemáticas, en el entorno del estudiante se mantienen los mitos y privilegios de la matemática Euro-Americana ${ }^{5}$. Una tercera

\footnotetext{
${ }^{5}$ Así llamada por Aikenhead (2017) con el propósito de resaltar sus raíces culturales.
} 
tensión interna se da en términos de distancia generacional, donde el conocimiento tradicional puede ser visto por los estudiantes como anticuado ya que estos experimentan la cultura de una manera diferente y, pueden sentir que esta perspectiva no es importante para ellos (Blanco, 2017; Nutti, 2013).

\section{Obstáculos}

Finalmente, se identifican algunos obstáculos señalados por los investigadores que dificultan las transformaciones en la política de educación matemática, propuestas desde el campo cultural, que se presentan en la Tabla 3, donde el nivel C se relacionó con las subcategorías alienación y colonialismo, el nivel B con la categoría complementariedad, y el nivel A con las categorías complementariedad y traducción:

\section{Tabla 3}

Obstáculos y consecuencias en relación con las subcategorías de análisis.

\begin{tabular}{|c|c|c|}
\hline Nivel & Obstáculo & Consecuencia \\
\hline $\begin{array}{l}\text { Principios } \\
\text { normativos }\end{array}$ & $\begin{array}{l}\text { Falta desarrollar la perspectiva } \\
\text { sociocultural de la educación } \\
\text { matemática en la escuela } \\
\text { (Aikenhead, 2017; Dos Santos y } \\
\text { Donizeti, 2011; Jaramillo, 2011; } \\
\text { Weldeana, 2016) } \\
\text { El discurso político de la } \\
\text { inclusión que al final termina } \\
\text { siendo una forma de neo- } \\
\text { colonialismo (Blanco et al., 2014; } \\
\text { Dos Santos y Donizeti, 2011; } \\
\text { Higuita, 2011; Tamayo, 2012). }\end{array}$ & $\begin{array}{l}\text { Impide abordar una historia no eurocéntrica } \\
\text { de las matemáticas e incluir saberes } \\
\text { extraescolares en el aula. Genera } \\
\text { dificultades de aprendizaje (alfabetización } \\
\text { matemática) y exclusión de creencias y } \\
\text { sistemas de valores. } \\
\text { Impide enfrentar el problema del } \\
\text { proyeccionismo. Genera exclusión y } \\
\text { discriminación; límites para el surgimiento } \\
\text { y desarrollo de nuevos paradigmas en la } \\
\text { educación matemática; presión sobre toda } \\
\text { la comunidad educativa por alcanzar } \\
\text { resultados altos en las pruebas } \\
\text { estandarizadas; homogenización y } \\
\text { mejoramiento de los aprendizajes. }\end{array}$ \\
\hline Institucional & $\begin{array}{l}\text { Desarticulación de la escuela con } \\
\text { la cosmovisión indígena (Costa, } \\
\text { 2012; Gavarrete, 2012; Jaramillo, } \\
\text { 2011; Tamayo, 2016; Weldeana, } \\
\text { 2016). }\end{array}$ & $\begin{array}{l}\text { Impide que los docentes tiendan puentes } \\
\text { interculturales y el fortalecimiento de la } \\
\text { cultura. Genera dificultades para la } \\
\text { alfabetización matemática y situaciones de } \\
\text { frontera. }\end{array}$ \\
\hline
\end{tabular}




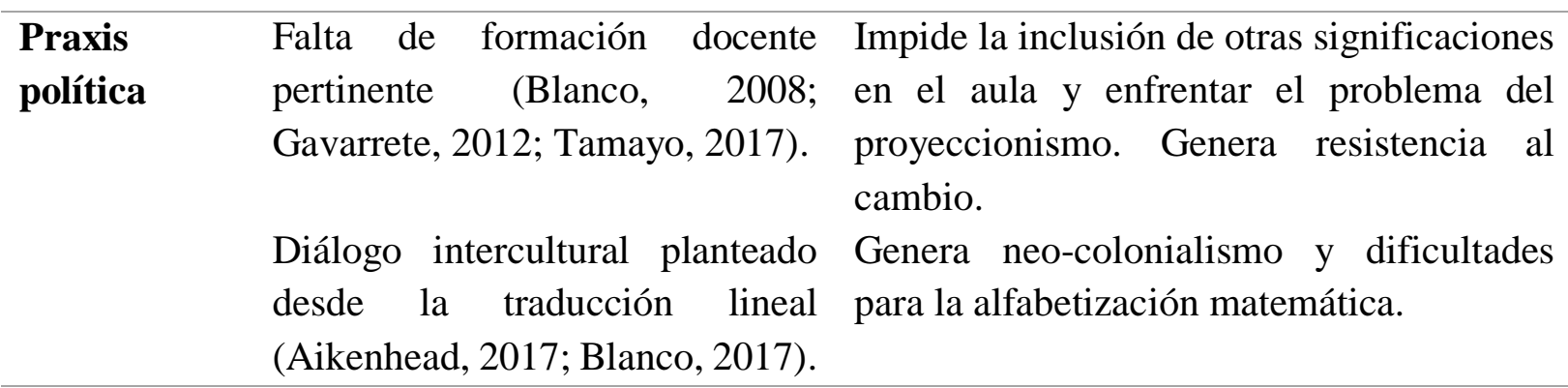

Fuente: producción propia.

\section{Conclusiones}

$\mathrm{Al}$ analizar la relación entre el currículo de matemáticas y la cultura indígena, se puede evidenciar un momento negativo o crítico y un momento positivo propositivo, donde lo cultural plantea transformaciones para la educación indígena, que fueron leídas desde los tres niveles de la política planteados por Dussel (2009), para lo cual se usaron las categorías construidas sobre la triple relación Educación Indígena - Currículo - Etnomatemática.

Respecto al momento negativo crítico, se asociaron las subcategorías colonialismo y alienación, mostrando cómo a través del control de los sujetos y el saber matemático, se generan procesos de ocultamiento y exclusión a partir del eurocentrismo y la modernidad.

Respecto al momento positivo, desde las categorías de emancipación, subjetividad, complementariedad, y traducción, se propone la criticidad del saber matemático, la reivindicación de otras racionalidades y de la relación con la Madre Tierra, la formación de estudiantes reflexivos, críticos, autónomos y fortalecidos en su identidad cultural, a partir de la complementariedad entre las prácticas culturales, la escuela y la comunidad, haciendo de la escuela un lugar de encuentro e hibridación cultural donde emerjan nuevos significados, sentidos, racionalidades, matemáticas y subjetividades, o en otras palabras un lugar donde se valore y promueva la diversidad.

En cuanto a las tensiones, estas se presentan en relación al cambio de una educación desde el colonialismo a una educación emancipadora, en la que la autonomía escolar se predica sin llegar a concretarse de acuerdo con las expectativas de las comunidades poniendo así a profesores y estudiantes en situaciones de frontera. 
Mientras que como obstáculos se señaló la falta de desarrollo de la perspectiva sociocultural de la educación matemática en la escuela, un falso discurso de inclusión, la desarticulación de la escuela con la cosmovisión indígena, la falta de formación adecuada de los profesores y un diálogo que se propone, pero no llega a ser verdaderamente intercultural.

Finalmente, se señala que las críticas y transformaciones que se plantean al currículo de matemáticas tienen como propósito último la resignificación de la educación matemática como una forma para el mejoramiento de la vida en sociedad desde el reconocimiento y valoración de la diversidad cultural, para vivir en armonía entre seres humanos sin ningún tipo de discriminación y en equilibrio con la naturaleza, lo cual no debería ser un propósito exclusivo de los pueblos indígenas sino de toda la humanidad puesto que como se ve en los acontecimientos actuales y como lo han denunciado muchos intelectuales como D’Ambrosio (2016) y Dussel (2015) nos encontramos ante la cercana posibilidad de la extinción de nuestra especie sobre la faz de la Tierra.

\section{Referencias}

(1) Aikenhead, G. S. (2017). Enhancing school mathematics culturally: a path of reconciliation. Canadian Journal of Science, Mathematics and Technology Education, 17(2), 73-140. https://doi.org/10.1080/14926156.2017.1308043

(2) Alves, T. (2017). Práticas docentes e o Programa Etnomatemática: o que revelam as pesquisas do ETNOMAT/RJ. Revista Latinoamericana de Etnomatemática, 10(3), 216-232.

(3) Arbelaez, J. \& Vélez, P. (2008). La etnoeducación en Colombia: una mirada indígena. Universidad EAFIT.

(4) Aroca, A. (2016). El Programa Etnomatemática: avances, desafíos y su papel en la globalización económica y el proyecto neoliberal. Revista Latinoamericana de Etnomatemática, 9(2), 238-277. http://www.redalyc.org/articulo.oa?id=274046804013

(5) Aroca, A. (2007). Una propuesta de enseñanza de geometría desde una perspectiva cultural. Caso de estudio: Comunidad Indígena Ika - Sierra Nevada de Santa Marta [Tesis de Maestría, Universidad del Valle]. http://etnomatematica.org/articulos/TesismaestriaAroca.pdf

(6) Asociación PANI. (2018). Proyecto para el fortalecimiento del pensamiento educativo propio: Asociación PANI (p. 10). 
(7) Avendaño, E., Díaz, L., Herrera, A., Higuita, C., Montoya, D. \& Quiceno, A. (2016). La Etnomatemática y la Educación Matemática: un recorrido epistemológico, curricular y metodológico en las investigaciones de la Universidad de Antioquia. Revista Latinoamericana de Etnomatemática, 9(1), 84-103.

(8) Barta, J. \& Shockey, T. (2016). Finding the 'space between' in developing mathematical curriculum and pedagogy across cultures: the mathematical Ways of the northern Ute. Journal of Mathematics and Culture, 10(3), 16-31.

(9) Blanco, H. (2009). Del número a los sistemas de numeración. Universidad del Valle.

(10)Blanco, H. (2017). Elementos para la formación de maestros de matemáticas desde la etnomatemática. Universidad de Granada.

(11)Blanco, H. (2008). La integración de la etnomatemática en la etnoeducación. Conferencia Presentada En $9^{\circ}$ Encuentro Colombiano de Matemática Educativa (16 Al 18 de Octubre de 2008), 7.

(12)Blanco, H., Fernández, A. \& Oliveras, M. (2017). Elementos de la formación de maestros desde la Etnomatemática que promueven la insubordinación creativa. In E. C. Lopez \& D. Jaramillo (Eds.), Escenas de la insubordinación creativa en las investigaciones en educación matemática en contextos de habla española (pp. 79-90). Raleigh: Lulu Press.

(13) Blanco, H., Ramírez, C. \& Oliveras, M. (2014). Una mirada a la Etnomatemática y la Educación Matemática en Colombia: caminos recorridos. Revista Latinoamericana de Etnomatemática, $\quad 7(2), \quad$ 245-269. http://www.revista.etnomatematica.org/index.php/RLE/article/view/130

(14) Cárdenas, M. \& Urueta, C. (2014). Diversidad cultural en la escuela pública colombiana 19602010. Educación y Ciudad, 26, 117-130. https://doi.org/10.36737/01230425.v.n26.2014.194

(15) Castro, S. (2005). La hybris del punto cero. Ciencia, raza e ilustración en la Nueva Granada (1750-1816) (Primera Ed). Editorial Pontificia Universidad Javeriana.

(16)CONTCEPI. (2013). Perfil del sistema educativo propio: SEIP. http://www.observatorioeducacion.org/images/Pdf/perfil-co.pdf

(17) Costa, L. (2009). Los tejidos y las tramas matemáticas. El tejido ticuna como soporte para la enseñanza de las matemáticas [Tesis de maestría, Universidad Nacional de Colombia]. http://www.bdigital.unal.edu.co/3309/

(18) Costa, L. (2012). A etnomatemática na educação do campo, em contextos indígena e 
ribeirinho, seus processos cognitivos e implicações à formação de professores [Tesis de maestría, Universdidad do estado do Amazonas]. http://repositorioinstitucional.uea.edu.br//handle/riuea/2578

(19) D'Ambrosio, U. (2016). The Ethnomathematics Program as a Proposal for Peace. International Journal for Research in Mathematics Education, 6(1), 8-25.

(20)Descartes, R. (1641). Meditaciones metafísicas. Escuela de Filosofía Universidad ARCIS. www.philosophia.cl

(21)Diaz, J. \& Batanero, C. (1994). Significado institucional y personal de los objetos matemáticos. Recherches En Didactique Des Mathématiques, 14(3), 325-335.

(22) Dos Santos, L. \& Donizeti, A. (2011). Educação Escolar Indígena, matemática e cultura: a abordagem etnomatemática. Revista Latinoamericana de Etnomatemática, 4(1), 21-39.

(23) Dussel, E. (2009). Política de la liberación. Volumen II: La arquitectónica (Primera ed). Editorial Trotta, S. A.

(24) Dussel, E. (2015). Filosofías del Sur (primera ed). Edicionesakal.

(25) Fuentes, C. (2014a). Descolonizando la escuela: ¿es posible llevar la Etnomatemática al aula? Revista Latinoamericana de Etnomatemática, 7(2), 222-244.

(26) Fuentes, C. (2014b). Etnomatemática, escuela y aprendizaje de las matemáticas: el caso de la comunidad de Guacamayas, Boyacá, Colombia. Universidad Francisco José de Caldas.

(27) Gavarrete, M. (2012). Modelo de aplicación de etnomatemáticas en la formación de profesores para contextos indígenas en costa rica. Universidad de Granada.

(28) Hammen, M. (1992). El manejo del mundo: Naturaleza y sociedad entre los Yukunas de la Amazonia colombiana (J. G. Saldarriaga \& T. van der Hammen (eds.); Segunda ed). TROPENBOS COLOMBIA.

(29) Higuita, C. (2014). La movilización de objetos culturales desde las memorias de la práctica de construcción de la vivienda tradicional Embera Chamí: posibilidades para pensar el (por)venir de la educación (matemática) indígena. Universidad de Antioquia.

(30) Higuita, C. (2011). La medida desde la medicina tradicional: el caso de una comunidad Embera Chamí. Universidad de Antioquia.

(31) Jaramillo, D. (2011). La educación matemática en una perspectiva sociocultural: tensiones, utopías, futuros posibles. Revista Educación y Pedagogía, 23(59), 13-36.

(32) Lacerda, N., Souza, M. \& Oliveira, R. (2017). A Matemática no currículo das escolas 
indígenas: um desafio da Educação Matemática. Revista Latinoamericana de Etnomatemática, 10(3), 149-166.

(33)Londoño, C. D. (2003). Muninane: un proyecto moral a perpetuidad (Primera ed). Editorial Universidad de Antioquia.

(34) Madrid, C. (2009). Filosofía de las Matemáticas: El cierre de la Topología y la Teoría del Caos. El Basilisco: Revista de Materialismo Filosófico, 41, 1-48.

(35) Miarka, R. (2011). Etnomatemática: do ôntico ao ontológico. Universidade Estadual Paulista.

(36) Ministerio de Educación Nacional. (1994). Ley general de educación 115 de 1994. Ministerio de Educación Nacional.

(37) Molano, E. \& Blanco, H. (2021). Revisión Documental Sobre La Relación Educación Indígena, Currículo Y Etnomatemática. Revista Amauta, En evaluación.

(38) Monteiro, A. \& Rodrigues, J. (2011). Prácticas sociales y organización curricular: cuestiones y desafíos. Revista Educación y Pedagogía, 23(59), 37-46.

(39) Monteiro, A. \& Rodrigues, J. (2014). A etnomatemática no encontro entre práticas e saberes: convergências, tensões e negociação de sentidos. Revista Latinoamericana de Etnomatemática, 7(3), 55-70.

(40) Nutti, Y. (2013). Sámi teachers' experiences of indigenous school transformation: culturally based preschool and school mathematics lessons. AlterNative: An International Journal of Indigenous Peoples, 9(1), 16-29. https://doi.org/10.1177/117718011300900102

(41) Nutti, Y. (2018). Decolonizing indigenous teaching: renewing actions through a Critical Utopian Action Research framework. Action Research, 16(1), 82-104. https://doi.org/10.1177/1476750316668240

(42) Parra, A. (2003). Acercamiento a la Etnomatemática. Universidad Nacional de Colombia.

(43) Parra, A. \& Trinick, T. (2017). Multilingualism in indigenous mathematics education: an epistemic matter. Mathematics Education Research Journal, 97-117. https://doi.org/https://doi.org/10.1007/s13394-017-0231-5

(44) Peña, P. \& Blanco, H. (2015). Reflexiones sobre cultura, currículo y etnomatemáticas. In R. Cortina \& K. de la Garza (Eds.), Educación, pueblos indígenas e interculturalidad en América Latina (Primera ed, pp. 213-245). Ediciones Abya-Yala.

(45)Peña, P., Blanco, H. \& Aroca, A. (2017). Incorporación de los conocimientos matemáticas indígenas en los sistemas educativos de Colombia y Chile (1990-2013). In E. Treviño, L. 
Morawietz, C. Villalobos, \& E. Villalobos (Eds.), Educación Intercultural en Chile: Experiencias, pueblos y territorios (pp. 261-302). Santiago de Chile: Ediciones UC, Editorial de la Pontificia Universidad Católica de Chile.

(46) Peña, P. A., Tamayo, C. \& Parra, A. (2015). Una visión latinoamericana de la etnomatemática: tensiones y desafíos. Revista Latinoamericana de Investigacion En Matematica Educativa, 18(2), 137-150. https://doi.org/10.12802/relime.13.1820

(47) Pereira, F., Oliveira, R. \& Santos, G. (2015). Concepções de matemática: do referencial curricular nacional para as escolas indígenas. Periferia Educação, Cultura e Comunicação, 7(1), 66-89.

(48) Pinxten, R. \& François, K. (2011). Politics in an Indian canyon? some thoughts on the implications of ethnomathematics. Educational Studies in Mathematics, 78(2), 261-273. http://www.jstor.org/stable/41486164

(49) Radford, L. (2013). Sumisión, alienación y (un poco de) esperanza: hacia una visión cultural, histórica, ética y política de la enseñanza de las matemáticas. In Y. Morales \& A. Ramirez (Eds.), Memorias del primer congreso de educación matemática de América Central y El Caribe (pp. 1-16).

(50) Rojas, A. \& Castillo, E. (2005). Educar a los otros: Estado, políticas educativas y diferencia cultural en Colombia (Primera Ed). Editorial Universidad del Cauca.

(51) Rosa, M. \& Orey, D. (2011). Ethnomathematics: the cultural aspects of mathematics. Revista Latinoamericana de Etnomatemática, 4(2), 32-54.

(52) Rosa, M. \& Orey, D. (2012). O campo de pesquisa em etnomodelagem: as abordagens êmica, ética e dialética. Educação e Pesquisa, 38(4), 865-879. https://doi.org/10.1590/S151797022012000400006

(53) Shockey, T. (2017). Confluence. Revista Latinoamericana de Etnomatemática, 10(1), 1-32. http://www.redalyc.org/articulo.oa?id=274048277010\%0AEsta

(54) Silva, B. \& Espasandin, C. (2015). Insubordinação Criativa: um convite à reinvenção do educador matemático. Bolema, 29(51), 1-17.

(55) Stathopoulou, C. \& Appelbaum, P. (2017). Polysemic provocations of border negotiation. ETD - Educação Temática Digital, $19(3), \quad$ 736-760. https://doi.org/10.20396/etd.v19i3.8648372

(56) Tamayo, C. (2012). (Re)significación del currículo escolar indígena, relativo al conocimiento 
[matemático], desde y para las prácticas sociales: el caso de los maestros indígenas Dule de la comunidad de Alto Caimán. Universidad de Antioquia.

(57) Tamayo, C. (2016). Currículo escolar, conocimiento [matemático] y prácticas sociales: posibilidades otras en una comunidad indígena Gunadule. Educação e Pesquisa, 42(4), 903919.

(58) Tamayo, C. (2017). Vení, vamos hamacar el mundo, hasta que te qsustes: uma terapia do desejo de escolarização moderna. Universidad Estadual de Campinas.

(59)Tutak, F., Bondy, E. \& Adams, T. (2011). Critical pedagogy for critical mathematics education. International Journal of Mathematical Education in Science and Technology, 42(1), 65-74. https://doi.org/10.1080/0020739X.2010.510221

(60) Villavicencio, M. (2011). Las etnomatemáticas en la educación intercultural bilingüe de Perú: avances y cuestiones a responder. XIII Ciaem-Iacme, 1-11. http://www.etnomatematica.org/publica/articulos/peru.pdf

(61) Weldeana, H. (2016). Ethnomathematics in Ethiopia: futile or fertile for mathematics education? Momona Ethiopian Journal of Science (MEJS), 8(2), 146-167. https://doi.org/http://dx.doi.org/10.4314/mejs.v8i2.4

Cómo citar este artículo: Molano, E. y Blanco, H. (2021). El currículo de matemáticas desde la cultura indígena: una revisión del estado del arte. Tendencias, 23(1), 423-448. https://doi.org/10.22267/rtend.222301.196 